\title{
Maintenance rehearsal and long-term recency
}

\author{
DEWEY RUNDUS \\ University of South Florida, Tampa, Florida 33620
}

\begin{abstract}
Maintenance (Type 1) rehearsal has been assumed to produce no increase in item recallability. Studies supporting this assumption have, however, tested limited ranges of the rehearsal time variable. The present study varied the time spent in Type 1 rehearsal over an extended range, and, with this added design sensitivity, recall was observed to be positively related to rehearsal duration. In addition, a pronounced long-term recency effect was observed. Both findings were interpreted as reflecting the role of contextual cues. An expanded description of the memorial product of Type 1 processing allows a preservation of the Type 1/Type 2 processing distinction and a reconciliation of the present and previous maintenance rehearsal data.
\end{abstract}

Craik and Lockhart (1972) called attention to the possibility that two quite different types of rehearsal activities might occur during item processing. They distinguished a Type 1 or maintenance rehearsal that consists of recycling already encoded information at some given processing level from a Type 2 processing that involves attempting to encode an item more deeply. These two classes of rehearsal activity were empirically distinguishable by the positive effect of Type 2 rehearsal on subsequent item recall and the absence of an enhancement of item recallability as a function of increasing duration of Type 1 rehearsal.

Since a positive relationship between amount of rehearsal and recall was a well accepted and widely observed phenomenon, attention was focused upon demonstrating the existence of Type 1 processing. Several early studies (e.g., Craik \& Watkins, 1973; Woodward, Bjork, \& Jongeward, 1973) indicated that if task instructions could induce subjects to hold an item in memory with no expectation of a subsequent test of memory for the item, then the duration of this maintain. ing activity had no reliable effect on the subsequent recallability of the item.

While these findings supported the existence of a type of rehearsal activity whose influence on recall was quantitatively quite different from the typical strong positive effect of rehearsal time on recall, it was not quite so obvious that the desired qualitative distinction could be assumed. Nelson (1977) described several problems that needed consideration prior to the acceptance of the Type $1 /$ Type 2 dichotomy. First, the relationship between the definition of Type 1 processing and the data confirming its existence is essentially circular. It is

A portion of these data was presented at the 1977 meeting of the Psychonomic Society. The author would like to thank Eliot Rifkin for his assistance in data collection and Susan Barrett and Jill Hekkanen for their helpful comments. Requests for reprints should be addressed to Dewey Rundus, Psychology Department, University of South Florida, Tampa, Florida 33620. only possible to know that Type 1 processing has occurred by observing a lack of positive correlation between rehearsal time and subsequent recall. Thus the noneffect of rehearsal on recall is not an observed but rather is a defined characteristic of maintenance rehearsal. Additionally, Nelson raised some procedural criticisms of the studies demonstrating Type 1 processing.

One approach to the problem of definitional circularity would be the demonstration of an experimental procedure in which rehearsal time could be manipulated and which would consistently demonstrate the absence of a rehearsal time effect on recall. It would then be legitimate to explore the characteristics of the type of rehearsal activity generated within that paradigm. The paradigm would thus serve as the operational definition of a particular type of rehearsal processing, and the influence of rehearsal time on recall could be taken as a characteristic of the rehearsal thus induced.

The distractor recall procedure, an incidental learning paradigm employed by Glenberg and Adams (1978), Glenberg, Smith, and Green (1977), and Rundus (1977) appears to satisfy the procedural objections posed by Nelson (1977). This procedure has produced data that, with the exception of one study (Rundus, 1977, Experiment 5), showed no significant relationship between the amount of time spent rehearsing an item and that item's subsequent recall. In this paradigm, subjects are led to believe that they are being tested for their short-term memory for numbers. Each Brown-Peterson type short. term memory trial consists of the presentation of a pair of two-digit numbers for study, overt processing of a word (usually simple repetition) serving as distractor activity for the number task, and finally, written recall of the studied digits. The distractor task is varied to provide different amounts of rehearsal and various types of processing of the word. At the completion of a series of these short-term memory trials, a filled temporal delay is introduced by requiring the subject to aid in the scoring of his number recall. Following this distraction, an unexpected free recall test for the rehearsed dis- 
tractor words is administered. The distractor recall procedure thus controls the subject's rehearsal activity by defining specific word processing tasks, ensures that the subject is processing each distractor word throughout its rehearsal interval by requiring overt rehearsal, and, typically, yields no relationship between recallability of an item on a delayed test and the amount of rehearsal time accorded that item.

Before concluding that the rehearsal activity produced by the distractor recall procedure really corresponds to the hypothesized Type 1 rehearsal, one final possibility needs to be considered. While previous research employing the distractor recall procedure does not seem to be subject to Nelson's (1977) criticism of a lack of statistical power, it is possible that the ranges of rehearsal time used thus far have not allowed adequate design sensitivity. Specifically, the rehearsal induced in this task may not be ineffective in incrementing longterm item recallability, as would be required if the processing really corresponded to the hypothesized Type 1 rehearsal, but merely inefficient. Long-term item recallability may increase with rehearsal time, but only gradually. Previous studies employing the distractor recall procedure have employed portions of the range of $4-18 \mathrm{sec}$ of rehearsal time per item. The study to be described employed a range of levels of the rehearsal time variable $(6-60 \mathrm{sec})$ that was expected to provide an adequately sensitive manipulation of rehearsal time.

\section{METHOD}

\section{Subjects}

The subjects were 32 undergraduate students from an introductory psychology class at the University of South Florida. They received extra credit toward their class grades for their voluntary participation.

\section{Material and Apparatus}

All stimuli were presented visually via an externally timed Kodak Carousel slide projector. Number stimuli were 20 pairs of two-digit numbers. Word stimuli were 20 medium-frequency nouns. All recall was written.

\section{Procedure}

The distractor recall procedure was utilized. The subjects were individually tested in a Brown-Peterson short-term memory task. Each subject was informed that the experiment involved a test of short-term memory for numbers. On each of 20 trials, the subject first was presented with a number slide displaying two two-digit numbers for 2 sec of silent study. The number slide was immediately replaced by a word slide showing a single word. The word slide was also presented for $2 \mathrm{sec}$. As soon as the word slide appeared, the subject began repeating the word over and over aloud at a moderate pace (one or two rehearsals/sec) and continued repeating the word after the slide disappeared and until the experimenter signaled for number recall. Each word was rehearsed overtly for a total of $6,18,36$, or 60 sec. A trial ended when the subject completed the written number recall and indicated to the experimenter to proceed to the next trial. Each trial involved presentation of a different pair of the to-beremembered numbers and a different word. After completion of all 20 trials, the subject read the correct answers to the shortterm memory number cover task from a key while the experi- menter scored the subject's response sheet. This task occupied about 1-1.5 min and served as interpolated distractor activity following the last study trial. The subject was then asked for written free recall of the distractor words that had been rehearsed during the Brown-Peterson trials. Following this unexpected free recall test, the subject was debriefed and questioned as to whether or not the test on the distractor words had been anticipated. Two subjects voiced a suspicion that recall of the distractor words might be required, and they were replaced by new subjects.

\section{Design}

A 4 (rehearsal time) by 5 (serial position) within-subjects design was employed. The four rehearsal times examined were $6,18,36$, and $60 \mathrm{sec}$. There were 20 short-term memory test trials that were balanced such that within subjects, each time occurred once in each serial position block, and across subjects, each time occurred once in each individual serial position. In addition, across subjects, each word occurred equally of ten in each serial position block at each level of the rehearsal time variable. The dependent variable was the number of words correctly recalled on the unexpected free recall test.

\section{RESULTS}

The probabilities of recall for distractor words on the free recall test are displayed in Table 1. A rejection region of $p<.05$ was used for all tests. The main effect of rehearsal time was significant $[F(3,93)=12.11$, $\mathrm{MSe}=.137]$. Inspection of the bottom row of Table 1 reveals a monotonic increase in recall as a function of rehearsal time. A significant main effect of serial position was also observed $[\mathrm{F}(4,124)=16.31, \mathrm{MSe}=.176]$. As may be seen in the right column of Table 1, there was a pronounced recency effect. The Rehearsal Time by Serial Position interaction was also significant $[F(12,372)=$ $1.94, \mathrm{MSe}=.143]$, with rehearsal time having the greatest effect in the recency portion of the curve.

\section{Type 1 Rehearsal and Recall}

Since the procedure employed in the present study was identical to that used by Rundus (1977) and differed only slightly (rehearsal was not paced) from the procedure of Glenberg and Adams (1978) and Glenberg et al. (1977), it appears that the rehearsal activities elicited by the distractor recall procedure may lead to at least a modest enhancement in subsequent item recallability. However, before concluding that the distractor recall procedure is not acceptable as a procedure that con-

Table 1

Probabilities for Free Recall for Distractor Words

\begin{tabular}{cccccc}
\hline & \multicolumn{4}{c}{ Rehearsal Time (in Seconds) } & \\
\cline { 2 - 5 } Position & 6 & 18 & 36 & 60 & Overall \\
\hline $1-4$ & .06 & .19 & .09 & .16 & .13 \\
$5-8$ & .03 & .13 & .13 & .22 & .13 \\
$9-12$ & .00 & .13 & .19 & .19 & .13 \\
$13-16$ & .22 & .13 & .34 & .47 & .29 \\
$17-20$ & .31 & .34 & .41 & .78 & .46 \\
Overall & .13 & .18 & .23 & .36 & \\
\hline
\end{tabular}


sistently induces rehearsal activities satisfying the strict Type 1 rehearsal requirement of no recall enhancement as a function of rehearsal time, it is appropriate to consider a further restriction on the procedure proposed by Glenberg and Adams (1978).

Glenberg and Adams (1978) proposed two additional criteria for determining whether maintenance rehearsal has been induced in the distractor recall procedure. They required that performance on the intentional cover task be off-ceiling (presumably indicating that the combined primary and secondary tasks were taxing the same resource) and that the level of primary task performance be constant for different durations of the secondary (incidental) task.

Specifically, they state, "If performance on the concurrent task is to be a valid indicator of the capacity devoted to the rehearsal task, then concurrent task performance must be off the ceiling because of a resource limitation imposed by the rehearsal task. ... And when performance on the concurrent task remains constant over an extended rehearsal period, we may conclude that the capacity devoted to the rehearsal did not change" (Glenberg \& Adams, 1978, p. 457).

In the present study, performance in the digit recall cover task was below ceiling, but digit recall performance was seen to decline as a function of time spent in distractor item rehearsal. The observed probabilities of correctly recalling both two-digit numbers on the shortterm digit recall task were $.91, .85, .84, .76$ for 6 -, 18-, 36-, and 60-sec distractor word rehearsal. The question that must be addressed is whether this observation serves as evidence that in the present study subjects were committing more than a necessary minimum of processing capacity to the rehearsed distractor items.

If performance on the concurrent (digit) task is to remain constant throughout the period of overt distractor word rehearsal, then we must assume that sufficient processing capacity is available to allow the distractor word rehearsal task to be noninterfering to the digit task. However, we also must observe below-ceiling performance on the digit task. Since, by assumption, the distractor word task is not producing interference with the digit task, we must seek some explanation for imperfect digit task performance. Possible sources of this below-ceiling performance might be problems in initial registration of the digit pairs or some test-specific output interference. With a 2 -sec digit presentation interval and only four digits to recall, neither of these possibilities seems compelling. It thus seems difficult to explain the off-ceiling digit task performance without assuming that distractor word rehearsal interferes with digit memory (and, consequently, expecting that digit task performance would be subject to at least some increasing interference from continued word rehearsal during the rehearsal interval).

Even if some other factor. not related to the rehearsal task, produces the less-than-perfect digit task performance, the existence of below-ceiling digit performance does not necessarily serve to indicate that the combined primary and secondary tasks fully occupy attention. For example, if something occurred during study or test (but not during distractor word rehearsal) that made it possible to recall only three of the four presented digits, then we are still ignorant of the total amount of processing capacity available during the rehearsal interval. Holding the three digits may occupy attention so fully that only the most minimal amount of processing capacity remains for dedication to the word task, or considerable attention may remain for allocation to the words. We cannot tell if only enough attention remained for minimal Type 1 word processing or if ample attention was available for Type 2 processing.

It seems, therefore, that if we really wish to limit the amount of processing that may be allocated to the word task, we should strive for a situation in which the subject must choose to restrict the attention given to word repetition in order to perform well on what the subject perceives to be the experimental task, the digit recall. This is perhaps best achieved by creating a situation in which even minimal allocation of attention to the word task will produce decrements in the concurrent digit task. In such a situation, it would be expected that the experimental requirement of overt word rehearsal would produce at least modest decrements in digit task performance throughout the rehearsal interval. It is, in fact, this need to induce allocation of some processing attention to the word task throughout the rehearsal interval (when such an allocation is perceived by the subject as probably detrimental to digit task performance) that underlies the requirement that the subject continue to repeat the distractor word overtly throughout the rehearsal interval. Without such a requirement, the subject would be highly unlikely to allocate resources needed for digit memory to the distracting word.

In summary, a case may be made that the Glenberg and Adams (1978) criteria for validating the occurrence of Type 1 rehearsal are not internally consistent. It must therefore be concluded that the results of the present study seriously undermine available empirical support for the existence of Type 1 rehearsal.

\section{Long-Term Recency}

One unexpected finding in the present study was an effect of serial position on recall. Due to the nature of the distractor recall procedure, serial position had not been examined previously. Multistore models have proposed that the differential probability of recall observed in the U-shaped serial position curve obtained in free recall tests is a result of the utilization of different memory stores; it is assumed that primacy items are recalled from long-term store and recency items are read out from short-term store (Atkinson \& Shiffrin, 
1968; Waugh \& Norman, 1965). It is argued that the enhanced recall of primacy items relative to midlist items is due to the primacy items' being accorded a greater amount of rehearsal. The finding that probability of recall increased monotonically with number of rehearsals (Rundus \& Atkinson, 1970) is cited as support for this argument. Since in the present study rehearsal of a word at any time other than during its presentation is unlikely due to the fact that word recall is unexpected, primacy should not occur. The data confirm this expectation.

The postulate that enhanced recall of recency items is due to readout from short-term store is supported by the finding that an interpolated task between study and free recall test eliminates the recency effect (Glanzer \& Cunitz, 1966). The distractor recall procedure includes an interpolated task prior to the free recall test of the distractor items; furthermore, subjects should not adopt a rehearsal strategy to maintain items in shortterm store because the free recall test is unexpected. Although the recency effect was thus not expected, it did appear in this experiment.

Other experiments have also produced serial position effects under circumstances in which a multistore model would have predicted no such effect. For example, in the continuous-distractor paradigm (Bjork \& Whitten, 1974; Tzeng, 1973), to-be-remembered items were assumed to be rehearsed equally, and a distractor task was interpolated between study and test; however, both primacy and recency effects were observed. Recency effects were also observed in incidental learning paradigms, in which the accumulation and readout of items in short-term store would not be expected (Marshall \& Werder, 1972; Postman \& Phillips, 1954).

Poltrock and MacLeod (1977) suggest that a multistore model can account for the U-shaped serial position curve in the continuous-distractor paradigm by asserting that the distractor task was unsuccessful in eliminating strategies of increased rehearsal of primacy items and readout from short-term store of recency items. The absence of the primacy effect in the present study, and in other experiments utilizing incidental paradigms, lends support to Poltrock and MacLeod's arguments that the primacy effect in the continuous-distractor paradigm results from a storage strategy (i.e., cumulative rehearsal of to-be-remembered items) adopted by the subject during intentional study. However, Poltrock and MacLeod's explanation of recency in the continuousdistractor paradigm does not seem to apply to the paradigm employed in the present study. Specifically, those rehearsed words that might have been in shortterm storage at the end of the study trials should have been lost due to the final interpolated task. Additionally, there is not reason to expect a subject to adopt any special strategy of retaining items in short-term store, since recall of the relevant items was not anticipated.

The significant Rehearsal Time by Serial Position interaction observed in the present data confirmed that time spent in continued repetition of a word was most beneficial for recency items. It is not clear, at present, whether this finding will aid in interpreting observed serial position effects, since this result seems compatible with current theories of long-term recency.

\section{CONCLUSION}

Since it appears that conclusive support for the existence of Type 1 rehearsal as defined by Craik and Lockhart (1972) is still to be found, it is perhaps appropriate to consider the motivation for that original definition. Craik and Lockhart were introducing the concept of processing depth and hypothesizing that it is the depth or qualitative type of code produced for an item that determines recall, and not the amount of time the item resides in short-term storage. This led to a contrast between a class of processing activities that was associated with attempts to encode an item more deeply and those activities that recycled or maintained already extracted codes. Since, according to Craik and Lockhart, processing depth was the ultimate determiner of recall, it seemed to follow naturally that the amount of time spent in maintenance (Type 1) processing should not enhance item recallability. This very reasonable assumption may prove to be too restrictive because, as the rather pronounced effects of code type on recall were emphasized, the potential contribution of contextual retrieval cues was overlooked.

When an internal code for an item is produced, that code may be associated by contiguity to the experimental context. To the extent that the experimental context remains constant during the maintenance in rehearsal of that code, no change in item retrievability via context cues should be observed. If the experimental context does change, either gradually or due to some specific environmental change, while a given item code is being maintained, then new context to code associations should be created. An increase in the number of context cues linked to an item code should provide more potential retrieval cues for the item and should enhance item recall in situations in which contextual cues are employed during retrieval.

In order to observe an increase in item memorability as a function of time spent in Type 1 processing, it would be necessary to examine a range of rehearsal times adequate to allow variations in context cues and to employ a test that is likely to induce the use of contextual cues during the test. The importance of the latter requirement is illustrated by the observation of effects of rehearsal times on recognition performance but not on free recall in studies in which the range of manipulated rehearsal times was fairly modest (e.g., Glenberg et al., 1977; Woodward et al., 1973). As an alternative to the use of very long rehearsal intervals (e.g., the present study) to allow context change, context change might be induced by presenting an item two separate times during a list, with the same level of processing required on each presentation. Recall of an item is strongly affected by 
number of presentations in both the distractor recall paradigm (Rundus, 1977) and other incidental tasks (e.g., McFarland, Rhodes, \& Frey, 1979; Nelson, 1977).

It seems, then, that a modest modification of assumptions could preserve the spirit of the original Craik and Lockhart (1972) dichotomy while reconciling the concept of Type 1 rehearsal to existent data. Type 2 rehearsal would label those rehearsal activities aimed at encoding an item more fully (i.e., deeper or more elaborate codes). Type 1 rehearsal would label the maintenance of already generated item codes, an activity that would benefit ultimate item memory to the extent that the item codes would be linked to variations in contextual cues that might occur during the maintenance period and to the extent that these contextual cues were employed in the memory test.

The hypothesized context to code associations may also underlie the long-term recency effects observed in the present study. The procedures employed in this experiment minimize the likelihood that the observed recency effects are due to short-term storage. While the present paucity of long-term recency data precludes a definitive explanation of the phenomenon, it is plausible to suggest that the effect reflects the subject's reliance primarily on contextual cues for retrieval. The absence of recency in delayed intentional free recall may be due to the subject's tendency in such a situation to rely primarily on interitem organization in attempting item retrieval (Baddeley, 1976, p. 185). In the present study, there is probably little or no interitem association formation; hence, subjects are left with the experimental context as the principal source of retrieval cues. If the experimental context is continually undergoing change, then the context cues present at test should be most similar to those present during the processing of the last items of the list and thus most likely to elicit those recency items.

The presence of long-term recency effects extending well back into an incidentally presented list should serve as a warning to researchers employing incidental paradigms. Care should be taken to balance within-list manipulations over serial positions to avoid potential confoundings.

\section{REFERENCES}

Atkinson, R. C., \& Shiffrin, R. M. Human memory: A pro- posed system and its control processes. In K. W. Spence \& J. T. Spence (Eds.), The psychology of learning and motivation: Advances in research and theory (Vol. 2). New York: Academic Press, 1968.

BADDELEY, A. D. The psychology of memory. New York: Basic Books, 1976.

BJORK, R. A., \& WhitTen, W. B. Recency-sensitive retrieval processes in long-term free recall. Cognitive Psychology, 1974, 6, 173-189.

Cralk, F. I. M., \& Lockhart, R. S. Levels of processing: A framework for memory research. Journal of Verbal Learning and Verbal Behavior, 1972, 11, 671-684.

Craik, F. I. M., \& Watkins, M. J. The role of rehearsal in short-term memory. Journal of Verbal Learning and Verbal Behavior, 1973, 12, 599-607.

Glanzer, M., \& Cunitz, A. R. Two storage mechanisms in free recall. Journal of Verbal Learning and Verbal Behavior, 1966, 5, 351-360.

Glenberg, A., \& Adams, F. Type I: Rehearsal and recognition. Journal of Verbal Learning and Verbal Behavior, 1978, 17, 455-463.

Glenberg, A., Smith, S. M., \& Green, C. Type I: Rehearsal, maintenance and more. Journal of Verbal Learning and Verbal Behavior, 1977, 16, 339-352.

McFarland, C. E., JR., Rhodes, D. D., \& Frey, T. J. Semanticfeature variability and the spacing effect. Journal of Verbal Learning and Verbal Behavior, 1979, 18, 163-172.

Marshall, P. H., \& Werder, P. R. The effects of the elimination of rehearsal on primacy and recency. Journal of Verbal Learning and Verbal Behavior, 1972, 11, 649-653.

Nelson, T. O. Repetition and depth of processing. Journal of Verbal Learning and Verbal Behavior, 1977, 16, 151-171.

Poltrock, S. E., \& MacLeod, C. M. Primacy and recency in the continuous distractor paradigm. Journal of Experimental Psychology: Human Learning and Memory, 1977, 3, 560-571.

Postman, L., \& Phillips, L. W. Studies in incidental learning: The effects of crowding and isolation. Journal of Experimental Psychology, 1954, 48, 48-56.

Rundus, D. Maintenance rehearsal and single-level processing. Journal of Verbal Learning and Verbal Behavior, 1977, 16, 665-681.

Rundus, D., \& Atkinson, R. C. Rehearsal processes in free recall: A procedure for direct observation. Journal of Verbal Learning and Verbal Behavior, 1970, 9, 99-105.

TzEnG, O. J. L. Positive recency effect in a delayed free recall. Journal of Verbal Learning and Verbal Behavior, 1973, 12, 436-439.

Waugh, N. C., \& Norman, D. A. Primary memory. Psychological Review, 1965, 72, 89-104.

Woonward, A. E., Jr., Bjork, R. A., \& Jongeward, R. H. $J_{R}$. Recall and recognition as a function of primary rehearsal. Journal of Verbal Learning and Verbal Behavior, 1973, 12, 608-617.

(Received for publication November 3, 1979; revision accepted February 4,1980 .) 